\title{
Tight coupling of soil quality with fungal community composition in a Chinese fir plantation chronosequence
}

\author{
Liang Chen ${ }^{1}$, Wenhua Xiang ${ }^{1}$, Shuai Ouyang ${ }^{1}$, Huili Wu ${ }^{1}$, Qi Xia ${ }^{2}$, Jiani Ma ${ }^{1}$, Yelin Zeng ${ }^{1}$, \\ Pifeng $\mathrm{Lei}^{1}$, wenfa xiao ${ }^{3}$, Shenggong $\mathrm{Li}^{4}$, and Yakov Kuzyakov ${ }^{5}$ \\ ${ }^{1}$ Central South University of Forestry and Technology \\ ${ }^{2}$ Central South University of Forestry and Technology Changsha Campus \\ ${ }^{3}$ Chinese Academy of Forestry \\ ${ }^{4}$ Institute of Geographical Sciences and Natural Resources Research, Chinese Academy of \\ Sciences \\ ${ }^{5}$ Institute of Physicochemical and Biological Problems of Soil Science. Russian Academy of \\ Sciences
}

April 28, 2020

\begin{abstract}
How soil quality and microbial communities change in conjunction with stand age in plantations is poorly understood. Here, we evaluated soil quality by using an integrated soil quality index (SQI) and traced the paralleled shifts in fungal community composition by high-throughput sequencing in a chronosequence of Chinese fir (Cunninghamia lanceolata) plantations (stand age of $3,16,25,32,>80$ years). Soil properties showed pronounced changes with stand age in the top $0-5 \mathrm{~cm}$. The most prominent increase from 3 to $>80$-year-old stand was for soil organic carbon (SOC, by 2.1-times), total nitrogen (TN, 1.9times) and available phosphorus (AP, 2.2-times). SQI increased logarithmically with stand age, with sharper change seen in the 0-5 cm layer than in the 5-15 cm layer. Mycorrhizal fungi increased in abundance initially in younger stands, but then they were gradually replaced by saprotrophic fungi in older stands due to the increase in litter input, which sustains saprotrophs. The positive correlation between saprotrophic fungi and the key soil quality indicators, such as TN, AP and NH4+, showed that higher soil quality was tightly linked with the enrichment of decomposers. Mycorrhizal taxa, such as orders Sebacinales, Thelephorales and Russulales, were positively correlated with acid phosphatase mobilizing P from organic matter. This suggests that the establishment of mycorrhizal fungi sustains tree productivity in younger stands under low soil quality. We conclude that the increase in soil quality throughout the development of Chinese fir plantations is closely linked with the observed transition of fungal communities from mycorrhizae to saprotrophs.
\end{abstract}

\section{Hosted file}

Manuscript .doc available at https : //authorea.com/users/291449/articles/440564-tight-couplingof-soil-quality-with-fungal-community-composition-in-a-chinese-fir-plantation-chronosequence 


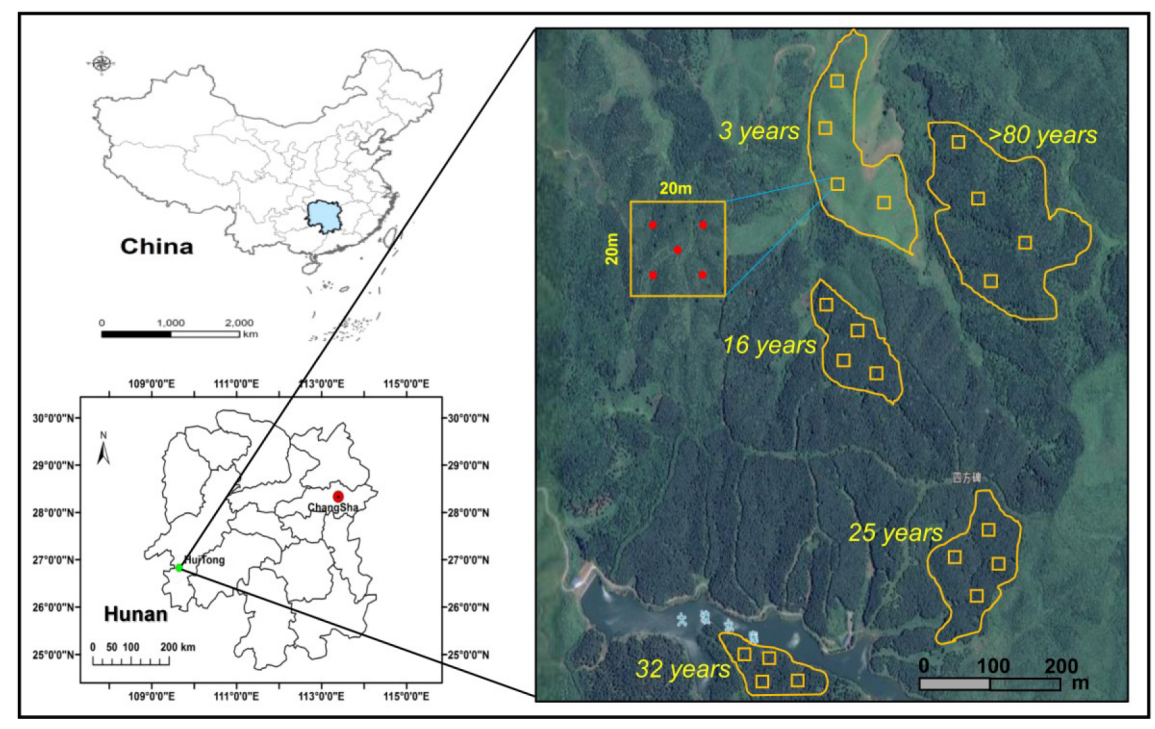



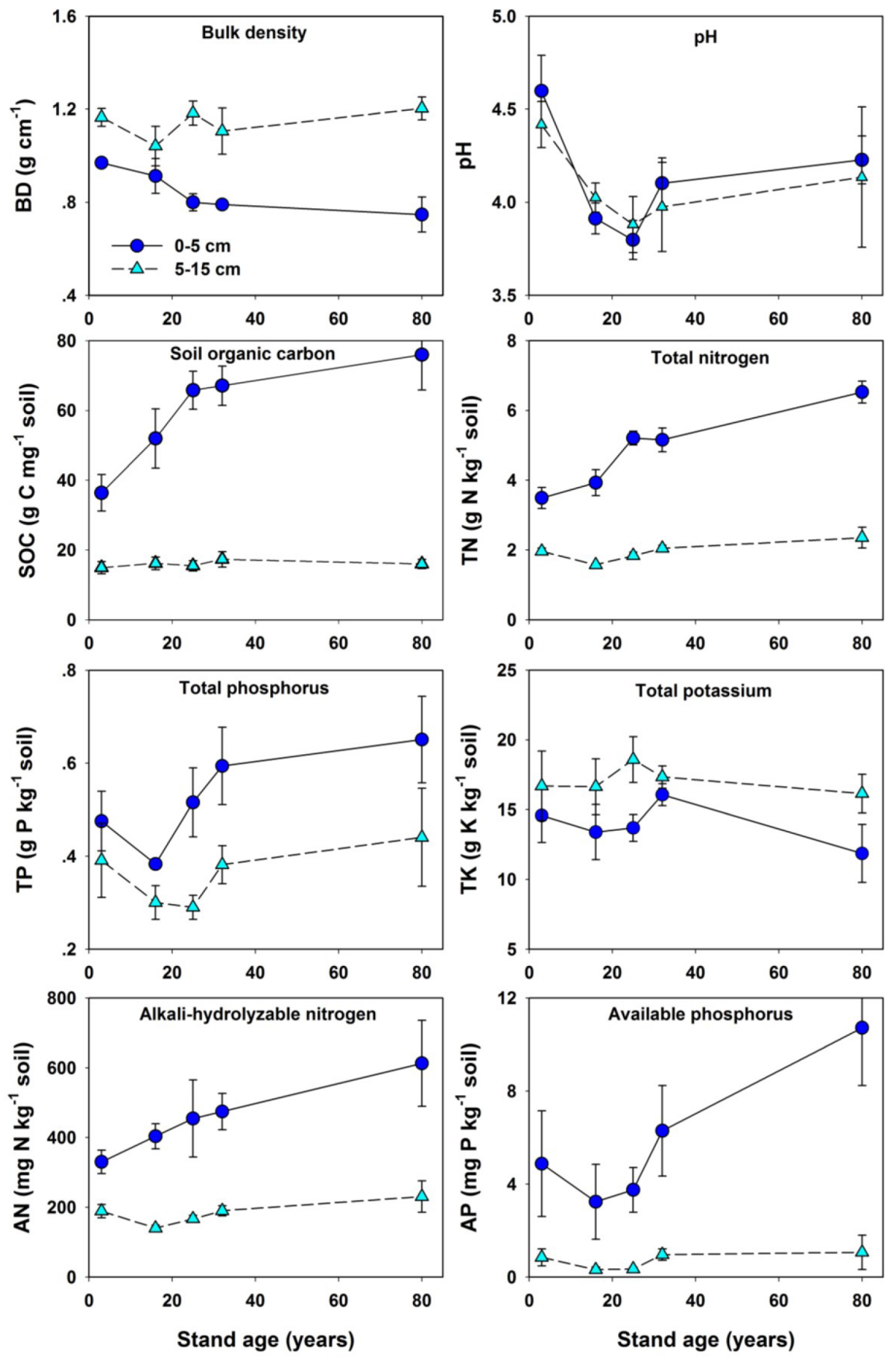

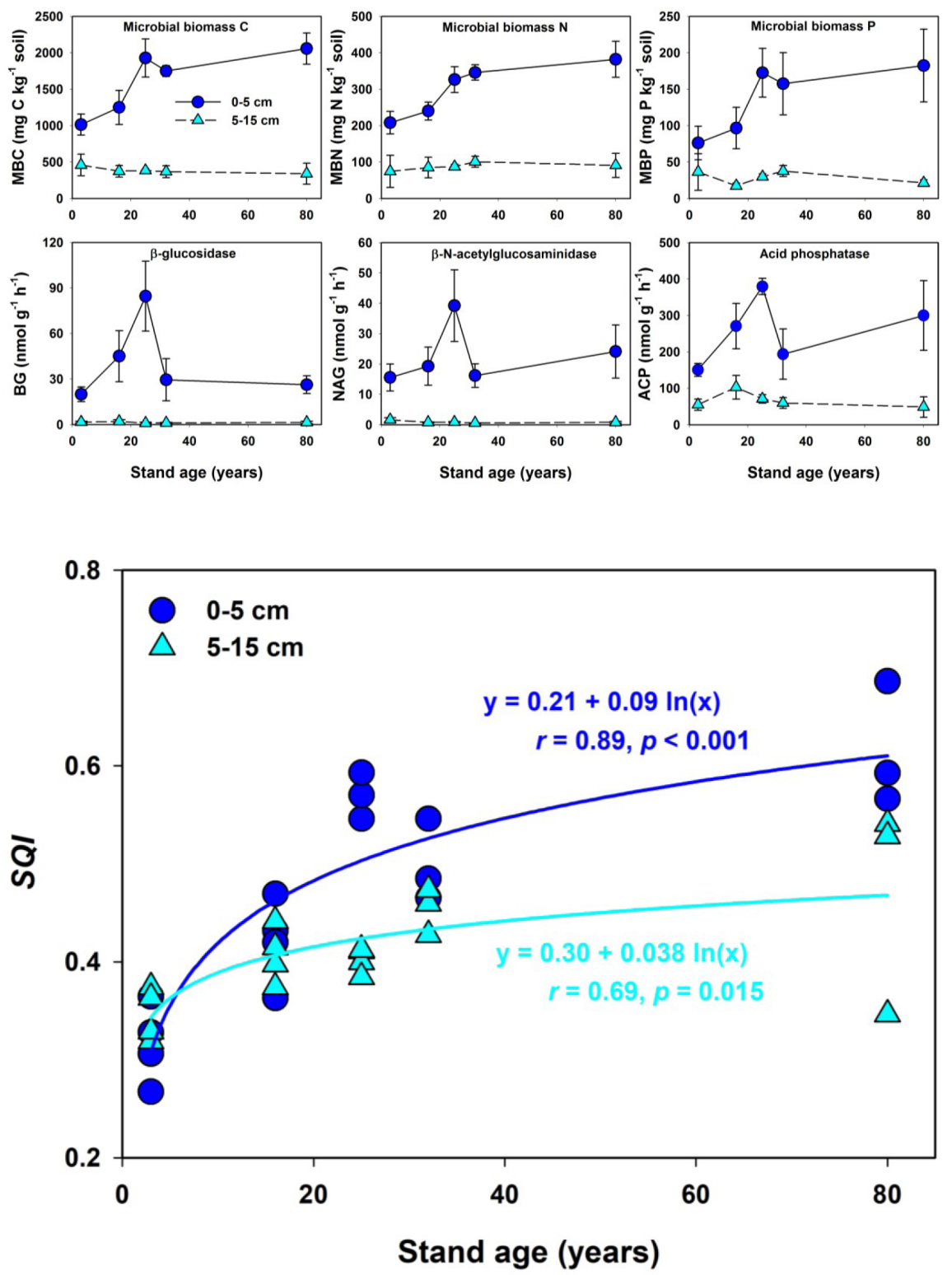

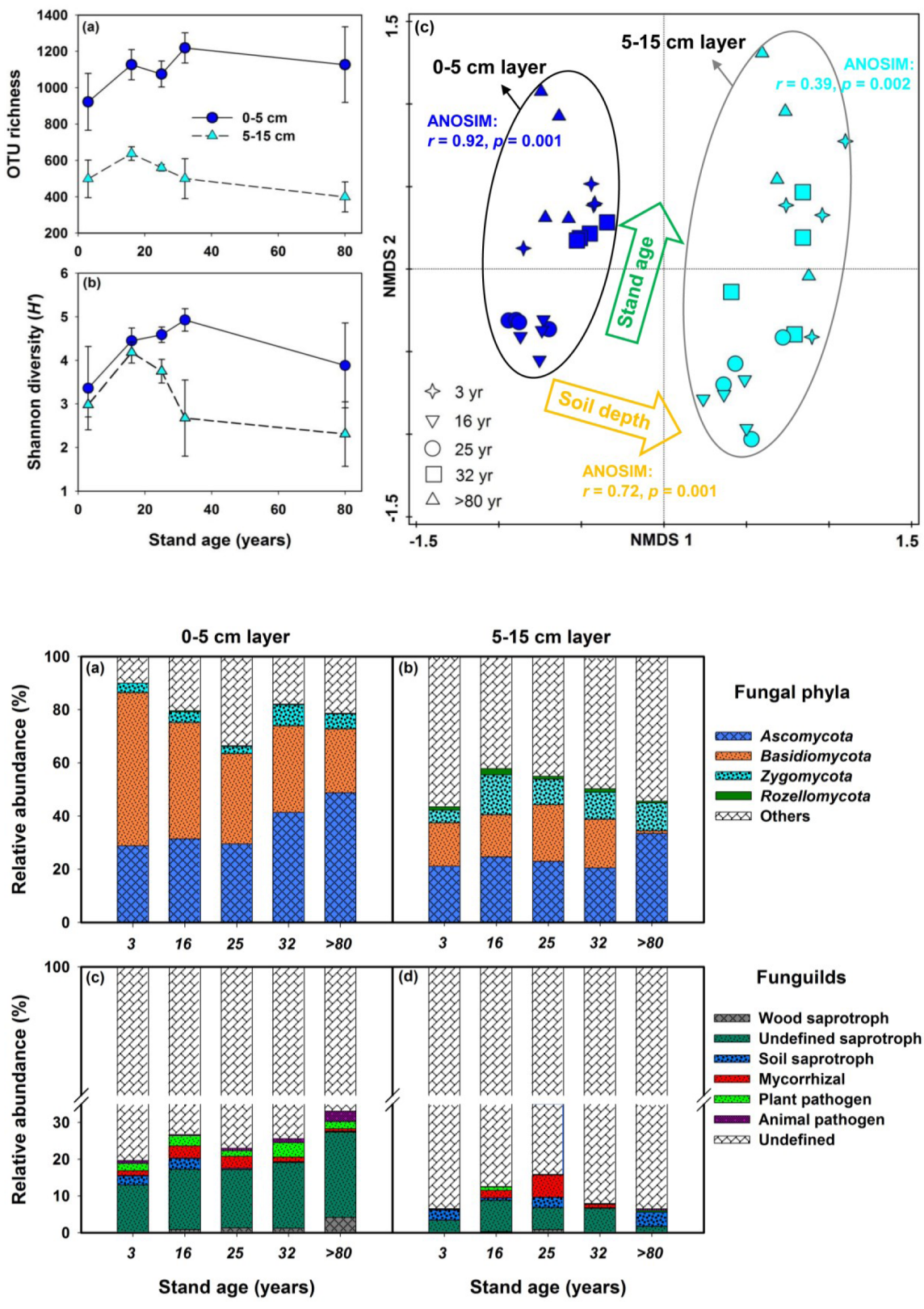

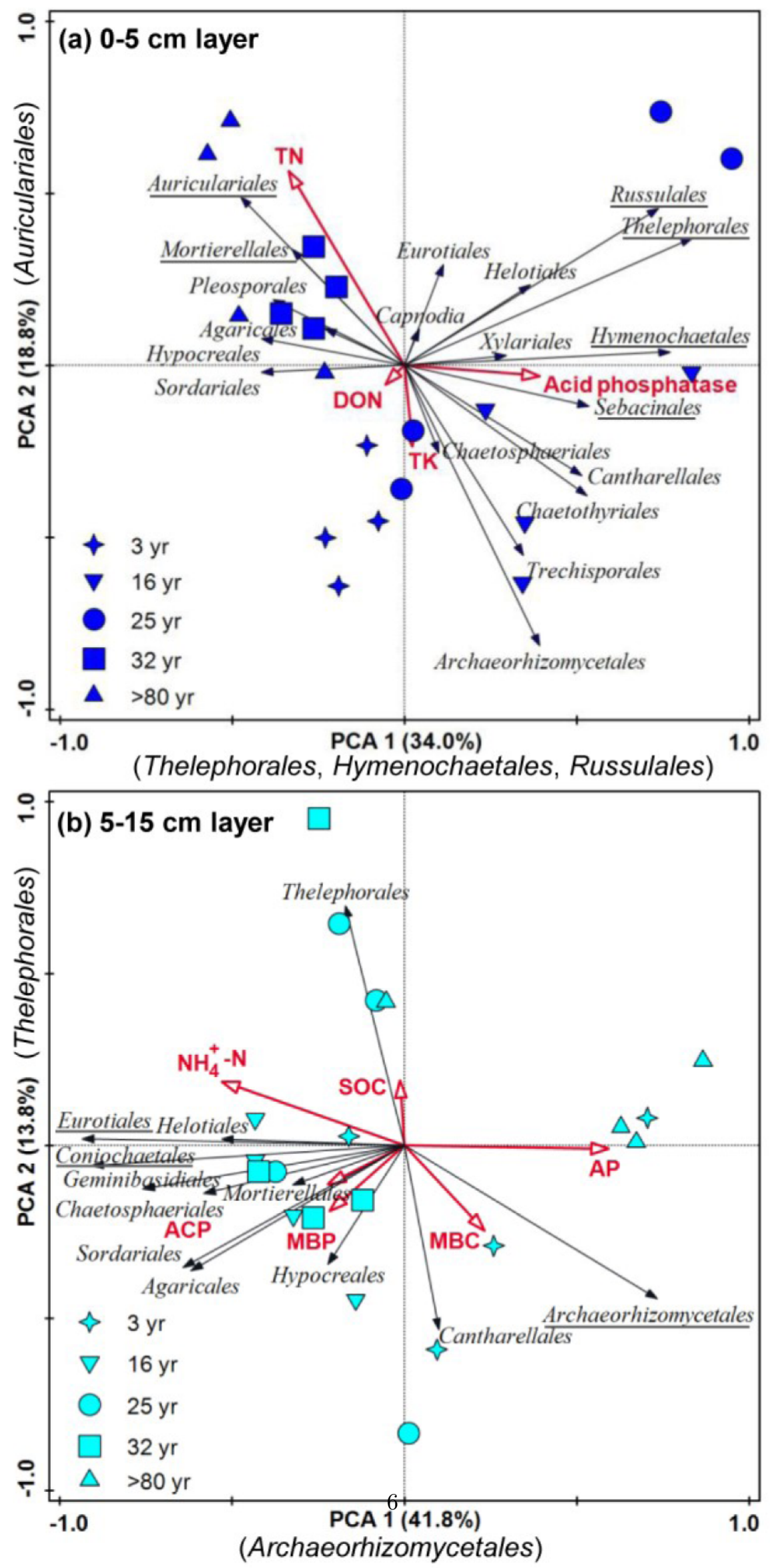


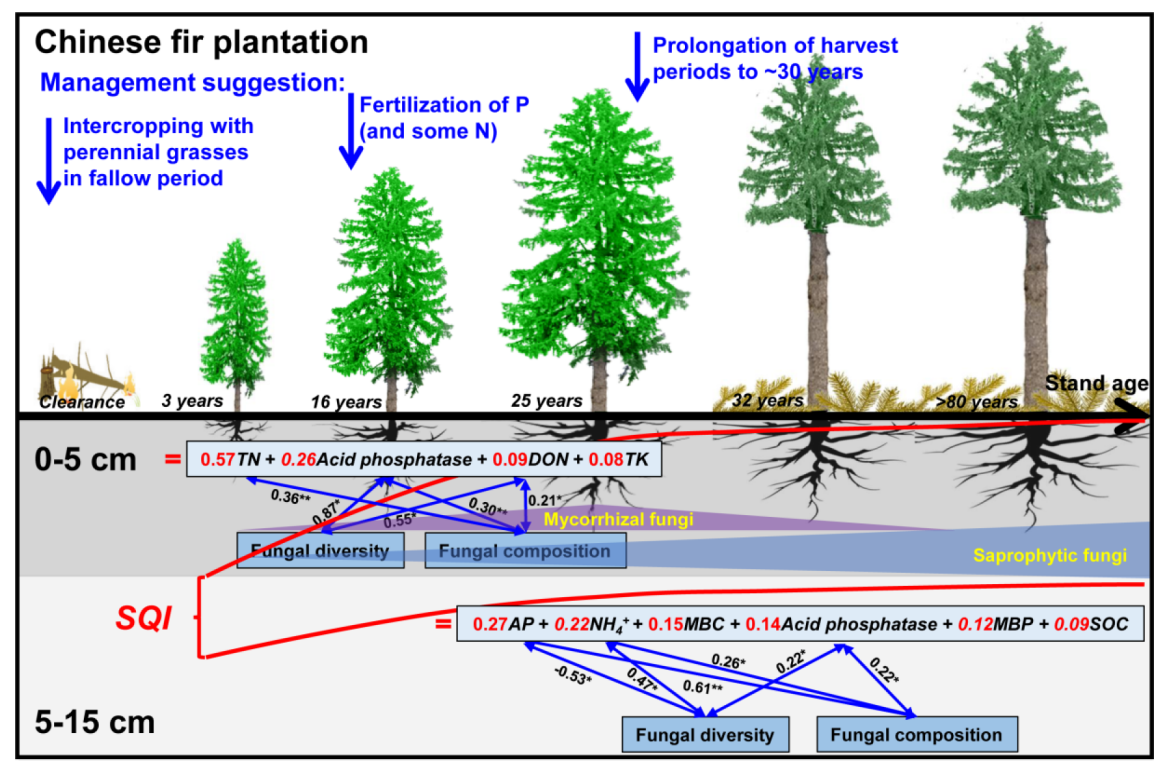

